\title{
Fluorescence-Based Molecular Imaging of Porcine Urinary Bladder Sentinel Lymph Nodes
}

\author{
Hak J. Lee ${ }^{1}$, Christopher V. Barback ${ }^{2,3}$, Carl K. Hoh ${ }^{2,3}$, Zhengtao Qin ${ }^{2,3}$, Kareem Kader ${ }^{1}$, David J. Hall ${ }^{2,3}$, David R. Vera ${ }^{2,3}$, \\ and Christopher J. Kane ${ }^{1}$ \\ ${ }^{1}$ Department of Urology, University of California, San Diego, La Jolla, California; ${ }^{2}$ Department of Radiology, University of \\ California, San Diego, La Jolla, California; and ${ }^{3}$ UCSD Molecular Imaging Program, University of California, San Diego, La Jolla, \\ California
}

\begin{abstract}
The primary objective was to test the ability of a laparoscopic camera system to detect the fluorescent signal emanating from sentinel lymph nodes (SLNs) approximately $2 \mathrm{~d}$ after injection and imaging of a positron-emitting molecular imaging agent into the submucosa of the porcine urinary bladder. Methods: Three female pigs underwent a submucosal injection of the bladder with fluorescent-tagged tilmanocept, radiolabeled with both ${ }^{68} \mathrm{Ga}$ and ${ }^{99 \mathrm{mTC}}$. One hour after injection, a pelvic PET/CT scan was acquired for preoperative SLN mapping. Approximately $36 \mathrm{~h}$ later, robotic SLN mapping was performed using a fluorescence-capable camera system. After identification of the fluorescent lymph nodes, a pelvic lymph node dissection was completed with robotic assistance. All excised nodal packets $(n=36)$ were assayed for $99 \mathrm{~m}$ Tc activity, which established a lymph node as an SLN. $99 \mathrm{~m} T$ Tc activity was also used to calculate the amount of dye within each lymph node. Results: All of the SLNs defined by the ex vivo $\gamma$-well assay of $99 \mathrm{mTc}$ activity were detected by fluorescence mode imaging. The time between injection and robotic SLN mapping ranged from 32 to $38 \mathrm{~h}$. A total of 5 fluorescent lymph nodes were detected; 2 pigs had 2 fluorescent lymph nodes and 1 pig exhibited a single lymph node. Four of the 5 SLNs exhibited increased SUVs of 12.4-139.0 obtained from PET/CT. The dye content of the injection sites ranged from 371 to $1,441 \mathrm{pmol}$, which represented $16.5 \%-64.1 \%$ of the injected dose; the amount of dye within the SLNs ranged from 8.5 to 88 pmol, which was equivalent to $0.38 \%-3.91 \%$ of the administered dose. Conclusion: Fluorescent-labeled ${ }^{68} \mathrm{Ga}$-tilmanocept allows for PET imaging and real-time intraoperative detection of SLNs during robotic surgery.
\end{abstract}

Key Words: fluorescence; molecular imaging; cystectomy; sentinel lymph node; pelvic lymph node dissection; robotic surgery; Lymphoseek; tilmanocept; PET/CT

J Nucl Med 2017; 58:547-553

DOI: 10.2967/jnumed.116.178582

A Pproximately 75,000 patients in the United States will be diagnosed and 16,000 will die from urinary bladder cancer (UBC) yearly. A quarter of patients diagnosed with UBC have muscle

Received Jun. 13, 2016; revision accepted Oct. 31, 2016.

For correspondence or reprints contact: David R. Vera, Moores UCSD Cancer Center, Molecular Imaging Program, 3855 Health Sciences Dr., La Jolla, California 92093-0819.

E-mail: dvera@ucsd.edu

Published online Feb. 2, 2017.

COPYRIGHT (C 2017 by the Society of Nuclear Medicine and Molecular Imaging. invasive bladder cancer (MIBC) accounting for $80 \%$ of the deaths due to UBC (1). Unfortunately, $25 \%$ of patients with MIBC have lymph node (LN) metastases at the time of cystectomy. If caught early, even MIBC has a good prognosis, with a 5- and 10-y recurrencefree survival rate after cystectomy for organ-confined disease of $89 \%$ and $78 \%$, respectively (2). However, if caught late the 5-y recurrence-free survival drops to less than $40 \%$ for those patients with node-positive UBC at cystectomy $(3,4)$.

The standard treatment for muscle invasive and select nonmuscle invasive disease is a radical cystectomy with an extended pelvic LN dissection (PLND) with consideration given to neoadjuvant chemotherapy. Several studies point to improved survival with a high-quality extended PLND compared with a limited type of PLND $(5,6)$.

However, the quality and extent of PLND in open and robotic cystectomy varies tremendously with the experience and volume of the surgeon performing the procedure $(7,8)$. Therefore, the identification of markers, which are able to elucidate relevant lymphatic tissue, would be clinically important and could possibly translate into improved surgical technique and also survival. Also, the potential for sentinel LN (SLN) identification for altering treatment management is significant if a future clinical trial confirms that negative SLN patients do not harbor metastases in downstream or secondary LNs. Future patients with negative SLNs could potentially be spared the costly, time-consuming, and potentially morbid extended lymphadenectomy, which is currently the standard of care for all patients undergoing cystectomy.

99mTc-tilmanocept (Lymphoseek; Cardinal Health) (9) is a radiopharmaceutical that accumulates in lymphatic tissue by avidly binding to a receptor that resides on the surface of macrophages (10) and dendritic cells (11). Lymphoseek has been approved in the United States and Europe for SLN mapping of breast cancer (12), melanoma (13), and head and neck cancer (14). A fluorescent-labeled version (15) of tilmanocept has been radiolabeled (16) with the positronemitting radionuclide ${ }^{68} \mathrm{Ga}$ and used to identify SLNs of the hind paw of rabbits (17) and prostate SLNs of dogs (18).

The ultimate goal of this project was to provide a new method of preoperative imaging and SLN identification during real-time robotic cystectomy. Therefore, using a porcine model, we sought to investigate the feasibility of a fluorescently tagged radiopharmaceutical for preoperative PET imaging $40 \mathrm{~h}$ before an extended robotic PLND. The role of the SLN in bladder cancer has been studied but has not been effectively tested for its potential benefits in MIBC. Therefore, from this study we hope to determine the feasibility of fluorescently tagged ${ }^{68} \mathrm{Ga}$-tilmanocept in a porcine model. If successful, we would proceed with a first-in-kind clinical 
trial for the eventual demonstration of clinical feasibility and effectiveness in patients with MIBC.

\section{MATERIALS AND METHODS}

\section{Experimental Design}

We designed a nonsurvival study using 3 female Yorkshire pigs to mimic a clinical protocol, which will include preoperative administration many days before robotic surgery with fluorescence-assisted SLN mapping before a complete LN dissection. The SLNs were detected by the molecular imaging agent tilmanocept, which was tagged with a near-infrared fluorescent dye, IRDye $800 \mathrm{CW}$, and radiolabeled with both ${ }^{68} \mathrm{Ga}$ and ${ }^{99 \mathrm{~m}} \mathrm{Tc} .{ }^{68} \mathrm{Ga}$ is a positron-emitting isotope with a 68 -min half-life, permitting imaging via PET, and ${ }^{99 \mathrm{~m}} \mathrm{Tc}$ is a $\gamma$-emitting isotope with a 6-h half-life. The covalent attachment of IRDye $800 \mathrm{CW}$ to tilmanocept is stable within SLNs for up to $72 \mathrm{~h}$ (16). ${ }^{99 m}$ Tc-tilmanocept is stable in human plasma for at least $11 \mathrm{~h}$ (16). Twelve LN packets from each of 3 animals yielded 36 possible accumulation sites by the fluorescent molecular imaging agent.

The primary goal of the study was to test the ability of the fluorescence camera system to detect all of the LNs that accumulated the molecular imaging agent. Ex vivo detection of ${ }^{99 m} \mathrm{Tc}$ activity within each SLN was the gold standard for SLN accumulation. The first of 2 secondary goals was to measure the amount of IRDye800 within each LN and investigate the ability of the FireFly camera system to detect pelvic LNs. An additional secondary goal was to explore the minimum injected dose of ${ }^{68} \mathrm{Ga}$ required for adequate PET/CT imaging. Both secondary goals were not designed to produce quantitative answers; their purpose was to guide the design of a phase 1 clinical trial.

First, using a cystoscope, we performed the submucosal injection into the bladder wall. One hour after injection, we performed hybrid PET/CT imaging of the pig's abdomen and pelvis. SUVs for each visible LN were calculated from the PET/CT images and used to determine whether the LN qualified as an SLN. Approximately $37 \mathrm{~h}$ after administration of the molecular imaging agent, the animal was anesthetized and instrumented using the da Vinci Si surgical robot (Intuitive Surgical) fitted with the FireFly endoscopic camera system. The 37-h interval between injection and surgery was selected to test the $\mathrm{LN}$ retention of the fluorescently tagged tilmanocept. With the use of preoperative PET/CT images for guidance, SLN mapping was performed via fluorescence imaging. After which, a complete extended PLND was performed, and during the dissection of each packet the FireFly imaging system was activated to observe fluorescent status of the packet. Each LN was defined as a true SLN if the ${ }^{99 \mathrm{~m}} \mathrm{Tc}$ activity was at least 3 times the SD of the background of the all $\gamma$-well-counter should be $\gamma$-well-counter and greater than $10 \%$ of the LN with the highest counts. This criterion permitted us to test the ability of the FireFly camera system to correctly identify each SLN. The ${ }^{99 \mathrm{~m}} \mathrm{Tc}$ activity also permitted us to calculate the amount of fluorophore accumulated within the LN.

\section{Animal Model}

Under a protocol approved by the Institutional Animal Care and Use Committee, sentinel node mapping for bladder cancer was evaluated in 3 adult female Yorkshire pigs $(30-35 \mathrm{~kg}$ ). All pigs were fasted overnight as per the protocol before each imaging and surgical procedure. The pigs were sedated by an intramuscular injection of an acepromazine/buprenorphine $(0.01-0.05$ and $0.01 \mathrm{mg} / \mathrm{kg}$ ) cocktail. After placement of a cephalic vein catheter, the animals were maintained under sedation by propofol infusion for the cystoscopy and radiopharmaceutical injection. The pig was awakened from the PET/CT procedure, taken to an approved holding area, and then brought to the operative suite to undergo general anesthesia for robotic surgery.

\section{Cystoscopy and Administration of Imaging Agent}

After anesthesia, we placed the pig supine and inserted the rigid cystoscope (Olympus $19 \mathrm{~F}, 30^{\circ}$ lens) into the urethra. After lubricated insertion of the cystoscope, the bladder was examined; the trigone and bilateral ureteral orifices were identified. We used a botox needle (Boston Scientific) to inject into the bladder under direct visualization. The single submucosal injection of ${ }^{68} \mathrm{Ga} /{ }^{99 \mathrm{~m}} \mathrm{Tc}-800 \mathrm{CW}$-tilmanocept (0.75 mL, $1.5 \mathrm{nmol}, 2.9-5.5 \mathrm{MBq}$ of ${ }^{68} \mathrm{Ga}, \sim 7.4 \mathrm{MBq}$ of ${ }^{99 \mathrm{~m}} \mathrm{Tc}, 1.5$ IRDye $800 \mathrm{CW}$ dyes per tilmanocept) was radiolabeled as previously described (16) and injected in the right trigone of the bladder, with care taken not to inject beyond the bladder wall. A wheal was visualized when injected at the correct depth. The bladder was drained with the cystoscope before image acquisition.

\section{PET/CT Image Acquisition and SUV-Based SLN Criteria}

Images of the injection site and pelvic LNs were acquired with a PET/CT system (Discovery VCT; GE Healthcare) using acquisition and processing protocols previously described $(18,19)$. A scout CT was acquired to confirm that the abdomen and pelvis were within the field of view. A low-dose CT scan (30-100 mA, $140 \mathrm{keV})$ was acquired for anatomic localization and attenuation correction. PET images (5 min, 3-dimensional mode, and isotope selection set to ${ }^{68} \mathrm{Ga}$; energy window of $375-650 \mathrm{keV}$ ) were acquired at $1 \mathrm{~h}$ after injection. Three bed positions were acquired to cover all the pelvic LN basins. Standard ordered-subset iterative reconstruction software (20 subset, 2 iterations) was used to generate attenuation-corrected transaxial images of $128 \times 128$ matrix size with $3.27-\mathrm{mm}$ slice thickness. Images were then reformatted when displayed as transaxial, coronal, and sagittal PET images with their corresponding cross-sectional CT images to produce fused PET/CT images. The SUV $\mathrm{max}_{\text {max }}$ within the region of interest of each visualized LN was obtained using the Volume Viewer software on an AW workstation (GE Healthcare). Any LN with an SUV that exceeded $10 \%$ of the highest LN SUV was defined as an SLN.

\section{Robotic Surgery and Fluorescence Imaging}

Robotic SLN mapping was performed on the second day after injection of the fluorescent-tagged radiopharmaceutical. After induction of anesthesia, insufflation was performed using a Veress needle, and subsequent port placement followed standard trocar placement for cystectomy. A $10-\mathrm{mm}$ camera trocar was placed in the midline, approximately $3 \mathrm{~cm}$ below the xyphoid, to visualize nodes that may be out of the usual field of view. A 10-mm assist port and 3 additional 8-mm robotic ports were placed in the standard robotic laparoscopic cystectomy distribution. The steep Trendelenburg position was used to allow the bowels to fall cephalad and away from the pelvis. The FireFly fluorescence imaging endoscope was used as a component of the da Vinci Si robotic surgical system (Intuitive Surgical). We reviewed the PET/CT scan for SLN localization and started the dissection at the bladder to confirm fluorescence at the injection site. Subsequently, we used the PET/CT to guide us to the region that contained an SLN and then used the FireFly fluorescence camera to confirm the SLN location in real time during the surgery. Using standard da Vinci Si system software, we switched from white light to near-infrared light to visualize the fluorescent LN. When the LN was found, we dissected the LN with robotic monopolar scissors. We then proceeded to complete the rest of the extended PLND up to the level of the inferior mesenteric artery. The following LN packets were excised and assayed for ${ }^{99 \mathrm{~m}} \mathrm{Tc}$ activity: the left and right external iliac, common iliac, perivascular, pelvic, and obturator LNs, as well as the presacral and paraaortic LNs. After completion of the robotic surgery, the animal was euthanized.

\section{Determination of SLN Dye Accumulation}

After dissection, each nodal packet and each dissected injection site were placed in a plastic scintillation vial and together with a counting 
standard were assayed for radioactivity using a $\gamma$-well-counter (100$200 \mathrm{keV}$ window; $\gamma$-9000 [Beckman Instruments]). The counting standard was a plastic scintillation vial containing a known fraction of the injectate. As previously described (18), we used the counts per minute (cpm) of the counting standard and each LN, the injected amount of tilmanocept $(1.5 \mathrm{pmol})$, and the average number of fluorescent dyes attached to each tilmanocept ( 1.5 dyes per tilmanocept) to calculate the number of fluorescent dye molecules accumulated by each LN and injection site. The background counting rate was determined for 10 empty scintillation vials.

The percentage injected dose was calculated in the following manner. We divided the ${ }^{99 \mathrm{~m}} \mathrm{Tc}$ activity of the $\mathrm{LN}$ or injection site by the activity of the counting standard. This result was multiplied by 100 and divided by the fraction of the injected dose within the counting standard.

\section{RESULTS}

Table 1 summarizes the independent and dependent variables pertaining to the primary objective of the study. For each SLN study, Table 1 lists the injection parameters of the molecular imaging agent as the amount of tilmanocept, the activity of ${ }^{68} \mathrm{Ga}$ and ${ }^{99 \mathrm{~m}} \mathrm{Tc}$, and the amount of dye administered. The elapsed time between PET/CT imaging and SLN mapping, the number of SLNs visualized by PET/CT, and the number of SLNs visualized by the FireFly camera are also listed for each study, as well as the number of LN packets excised and the number of SLNs detected ex vivo by the $\gamma$-counter assay of ${ }^{99 \mathrm{~m}} \mathrm{Tc}$ activity. Of the independent variables, the amounts of tilmanocept and IRDye $800 \mathrm{CW}$ dye were held constant at 1.5 and $2.25 \mathrm{nmol}$, respectively. The ${ }^{99 \mathrm{~m}} \mathrm{Tc}$ dose ranged from 7.4 to $8.9 \mathrm{MBq}$, which was more than adequate for the $\gamma$-well-counter to measure tilmanocept uptake within the SLN $48 \mathrm{~h}$ after administration. The amount of ${ }^{68} \mathrm{Ga}$ injected ranged from 5.5 to $2.9 \mathrm{MBq}$; the lowest dose provided an opportunity to explore the minimum activity within the SLN that could be detected by the PET/CT scanner. The time between administration and surgery ranged from 33 to $39 \mathrm{~h}$.

Both preoperative PET/CT imaging and intraoperative fluorescence mapping identified at least 1 SLN during each of the studies. When the $10 \%$ rule was used, preoperative SUVs identified an additional SLN during 2 of the PET/CT imaging studies. When the preoperative PET/CT scans for regional location were used, the fluorescent LNs were encountered within minutes of dissection. All PET/CT-detected SLNs (100\%) were identified by the FireFly camera system during fluorescence mapping. Of the fluorescent nodes visualized by the camera system, 4 of $5(80 \%)$ satisfied the $10 \%$ rule defined by the PET/CT scan.

Table 2 pertains to the secondary study objectives. It lists the LN and injection site SUVs at the time of PET/CT imaging and whether the SUV satisfied the $10 \%$ rule for qualification as the SLN. Also listed are the fluorescence status of each LN percentage injected dose and dye content values for each of the LN packets and the dissected injection site of each study. The SUV entry for a given LN is listed, only if the LN was not visualized by PET/CT or if the ${ }^{99 \mathrm{~m}} \mathrm{Tc}$ measurement by the $\gamma$-counter was greater than the mean $(\bar{x}=24.6 \mathrm{cpm})$ plus $3 \mathrm{SDs}(\mathrm{SD}=5.1 \mathrm{cpm})$ of the background counting rate, which equaled $39.9 \mathrm{cpm}$. The SUVs ranged from 12.4 to 139.0 for the LNs and 1,769 to 20,322 for the injection sites. The percentage injected dose and dye content entries are blank if the $\gamma$-counter measurement for a given $\mathrm{LN}$ or injection site was less than $39.9 \mathrm{cpm}$. Of the LNs that measured greater than $39.9 \mathrm{cpm}$, the percentage injected dose ranged from $0.38 \%$ to $3.91 \%$, and the dye content, reported in pmol of IRDye $800 \mathrm{CW}$, ranged from 0.89 to 88.0 pmol. The percentage injected dose remaining in the injection sites at the time of SLN mapping ranged from $16.5 \%$ to $64.1 \%$, and the dye content ranged from 371 to $1,441 \mathrm{pmol}$ of IRDye800CW.

We provide study two as an example to demonstrate the sequence of events. We injected the fluorescent radiopharmaceutical $\left(4.4 \mathrm{MBq}\right.$ of ${ }^{68} \mathrm{Ga}, 7.4 \mathrm{MBq}$ of ${ }^{99 \mathrm{~m} T \mathrm{~T},}, 1.5 \mathrm{nmol}$ of tilmanocept, $2.25 \mathrm{nmol}$ of fluorescent dye) into the submucosa of the right trigone of the bladder and created a wheal with a faint blue color (Fig. 1). After a scout CT, a 3-bed-position PET/CT of the pelvis was acquired $1 \mathrm{~h}$ after injection. Only a right perivesicular LN was detected by PET/CT (SUV = 65.2); the injection site had an SUV of 1,769. The transaxial (Fig. 2A) and coronal (Fig. 2B) crosssections demonstrate the ability of PET/CT to clearly discriminate the perivesicular LN from the adjacent injection site. Figure $2 \mathrm{C}$ is a sagittal view of the right perivesicular LN. At the time of these images, the $\mathrm{LN}$ contained approximately $0.085 \mathrm{MBq}$ of ${ }^{68} \mathrm{Ga}$. The pig was recovered, returned to the vivarium, and $2 \mathrm{~d}$ later brought to the surgical research suit and instrumented with a da Vinci Si surgical robot equipped with a FireFly fluorescence-capable endoscopic camera system. Robotic LN dissection (Supplemental Video 1; supplemental materials are available at http://jnm. snmjournals.org) started $36 \mathrm{~h}$ after injection of the fluorescentlabeled tilmanocept. The bladder was mobilized to investigate the injection site and confirm fluorescence. On the basis of the preoperative $\mathrm{PET} / \mathrm{CT}$ as guidance, the right perivesicular

TABLE 1

Primary Objective: Dose Parameters and Number of Lymph Nodes Excised and Detected

\begin{tabular}{|c|c|c|c|c|c|c|c|c|}
\hline \multirow[b]{2}{*}{ Study no. } & \multicolumn{4}{|c|}{ Molecular imaging agent } & \multirow{2}{*}{$\begin{array}{l}\text { Time between } \\
\text { administration and } \\
\text { SLN mapping (h) }\end{array}$} & \multirow{2}{*}{$\begin{array}{l}\text { No. of SLNs } \\
\text { visualized by } \\
\text { the endoscopic } \\
\text { camera }(n)\end{array}$} & \multirow[b]{2}{*}{$\begin{array}{l}\text { Lymph nodes } \\
\text { excised }(n)\end{array}$} & \multirow{2}{*}{$\begin{array}{l}\text { No. of SLNs } \\
\text { detected ex } \\
\text { vivo by } \\
\text { y-counting* }(n)\end{array}$} \\
\hline & $\begin{array}{l}\text { Tilmanocept } \\
\text { (nmol) }\end{array}$ & $\begin{array}{l}{ }^{68} \mathrm{Ga} \\
(\mathrm{MBq})\end{array}$ & $\begin{array}{l}\text { 99mTc } \\
(\mathrm{MBq})\end{array}$ & $\begin{array}{l}\text { IRDye } 800 \mathrm{CW} \\
\text { dye (nmol) }\end{array}$ & & & & \\
\hline One & 1.5 & 5.5 & 8.1 & 2.25 & 38 & 2 & 12 & 2 \\
\hline Two & 1.5 & 4.4 & 7.4 & 2.25 & 36 & 1 & 12 & 1 \\
\hline Three & 1.5 & 2.9 & 8.9 & 2.25 & 32 & 2 & 12 & 2 \\
\hline
\end{tabular}

${ }^{*}$ Using $10 \%$ rule based on ${ }^{99 m}$ Tc radioactivity in cpm. 
TABLE 2

Secondary Objectives: SLN and Injection Site Accumulation During PET/CT and Visualization During Robotic Surgery

\begin{tabular}{|c|c|c|c|c|c|c|c|c|c|c|c|c|c|c|c|}
\hline \multirow[b]{3}{*}{$\begin{array}{c}\text { Lymph } \\
\text { node/injection } \\
\text { site }\end{array}$} & \multicolumn{5}{|c|}{ Study one } & \multicolumn{5}{|c|}{ Study two } & \multicolumn{5}{|c|}{ Study three } \\
\hline & \multicolumn{2}{|c|}{ PET/CT } & \multirow[b]{2}{*}{$\begin{array}{c}\text { FireFly } \\
\text { in vivo } \\
\text { fluorecence? } \\
\text { (yes/no) }\end{array}$} & \multicolumn{2}{|c|}{$y$-counter } & \multicolumn{2}{|c|}{$\mathrm{PET} / \mathrm{CT}$} & \multirow[b]{2}{*}{$\begin{array}{c}\text { FireFly } \\
\text { in vivo } \\
\text { fluorescence? } \\
\text { (yes/no) }\end{array}$} & \multicolumn{2}{|c|}{$\mathrm{y}$-counter } & \multicolumn{2}{|c|}{$\mathrm{PET} / \mathrm{CT}$} & \multirow[b]{2}{*}{$\begin{array}{c}\text { FireFly } \\
\text { in vivo } \\
\text { fluorescence? } \\
\text { (yes/no) }\end{array}$} & \multicolumn{2}{|c|}{$y$-counter } \\
\hline & SUV & $\begin{array}{c}\text { Satisfy } \\
10 \% \text { rule? } \\
\text { (yes/no) }\end{array}$ & & $\begin{array}{l}\text { Percentage } \\
\text { injected } \\
\text { dose }(\%)\end{array}$ & $\begin{array}{l}\text { Dye } \\
\text { content } \\
\text { (pmol) }\end{array}$ & t & $\begin{array}{c}\text { Satisfy } \\
10 \% \\
\text { rule? } \\
\text { (yes } / \text { no) }\end{array}$ & & $\begin{array}{l}\text { Percentage } \\
\text { injected } \\
\text { dose }(\%)\end{array}$ & $\begin{array}{c}\text { Dye } \\
\text { content } \\
\text { (pmol) }\end{array}$ & - & $\begin{array}{c}\text { Satisfy } \\
10 \% \\
\text { rule? } \\
\text { (yes/no) }\end{array}$ & & $\begin{array}{l}\text { Percentage } \\
? \quad \text { injected } \\
\text { dose }(\%)\end{array}$ & $\begin{array}{l}\text { Dye } \\
\text { content } \\
\text { (pmol) }\end{array}$ \\
\hline $\begin{array}{l}\text { L. external } \\
\text { iliac }\end{array}$ & & & No & & & & & No & & & & & & & \\
\hline $\begin{array}{l}\text { R. external } \\
\text { iliac }\end{array}$ & & & No & & & & & No & & & NV & & Yes & 0.38 & 8.50 \\
\hline Presacral & & & No & & & & & No & & & & & No & & \\
\hline Para aortic & & & No & & & & & No & & & & & No & & \\
\hline $\begin{array}{l}\text { L. common } \\
\text { iliac }\end{array}$ & & & No & & & & & No & & & & & No & & \\
\hline $\begin{array}{l}\text { R. common } \\
\text { iliac }\end{array}$ & 12.4 & Yes & Yes & 2.72 & 32.3 & & & No & & & 139 & Yes & Yes & 1.33 & 29.8 \\
\hline L. perivesicular & & & No & & & & & No & & & & & No & & \\
\hline R. perivesicular & 59.0 & Yes & Yes & 1.44 & 61.0 & 65.2 & Yes & Yes & 3.91 & 88.0 & & & No & & \\
\hline L. pelvic & & & No & & & & & No & & & & & No & & \\
\hline R. pelvic & & & No & & & & & No & & & & & No & & \\
\hline L. obturator & & & No & & & & & No & & & & & No & & \\
\hline R. obturator & & & No & & & & & No & & & & & No & & \\
\hline Injection site & 2,911 & & Yes & 29.2 & 658 & 1,769 & & Yes & 16.5 & 371 & 20,322 & & Yes & 64.1 & 1,441 \\
\hline
\end{tabular}

LN was identified with brightfield illumination (Fig. 3A) and its intense fluorescence noted (Fig. 3B). None of the remaining LN packets exhibited fluorescence in vivo under FireFly illumination. After dissection, all of the LN packets as well as the injection site were examined ex vivo for identification of fluorescence and assayed for ${ }^{99 \mathrm{~m}} \mathrm{Tc}$ activity, permitting us to calculate the amount of fluorescent dye accumulated by the 12 LNs excised. Only the right perivesicular LN was fluorescent. The radioactivity assay demonstrated 88.0 pmol of dye in this LN and 371 pmol within the injection site. The remaining LNs contained less than 0.1 pmol of dye, which corresponded to the detection limit of the $\gamma$-counter.

\section{DISCUSSION}

This study, based on examination of $36 \mathrm{LNs}$, demonstrated that the FireFly fluorescence imaging system is capable of detecting fluorescent-labeled tilmanocept up to $38 \mathrm{~h}$ after a submucosal injection into the porcine urinary bladder. This result is consistent with fluorescence imaging of SLNs $36 \mathrm{~h}$ after fluorescent tilmanocept injection into the footpad of rabbits (20) and the canine prostate gland (18).

Our previous work with the rabbit model (20) demonstrated the ability of the FireFly system to detect fluorescent tilmanocept using an injected dose that would not saturate the receptor sites within the LN. The experiments that described this current work used an animal model specific to bladder wall SLN mapping. We used injections into the submucosal layer of the bladder wall, which has significantly different lymphatic drainage than the foot- pads of the rabbit. Although the previous study (20) used the same fluorescence imaging system/camera, the pelvic LNs that drain the bladder wall are embedded within a layer of fat that envelopes major blood vessels or nerves, an anatomic setting that differs greatly from rabbit popliteal LNs that reside between muscle layers. As a result, the animal model used in the current work provides a far more realistic and demanding challenge to successful fluorescence imaging.

Detection for SLN has been an established technique in breast and melanoma surgery; however, the technique is not the standard of care and only considered experimental in bladder cancer. Research has examined the possibility of the SLN in UBC. From past anatomic studies, we understand that $74 \%, 65 \%$, and $19 \%$ of sites of nodal metastasis involved obturator/hypogastric, internal iliac, and common iliac LNs, respectively, during cystectomies (21). However, there have been only few clinical studies looking into mapping and detection of SLNs (22), the role of SLNs in improving nodal staging (23), and the role of SLNs in early metastatic progression (24).

It is obvious that with the use of radiolabeled or fluorescencelabeled tilmanocept, one of the major clinical benefits would be to identify the SLN intraoperatively in real time and to ensure that the SLN is not left behind when performing PLND during cystectomy. In an SLN study by Sherif et al., for 13 patients who underwent radical cystectomy, 4 were found to have metastases; all 4 demonstrated positive sentinel nodes, and there were no false-negative SLNs (22). Furthermore, Dangle et al. demonstrated that when commonly performed templates for node dissection in 


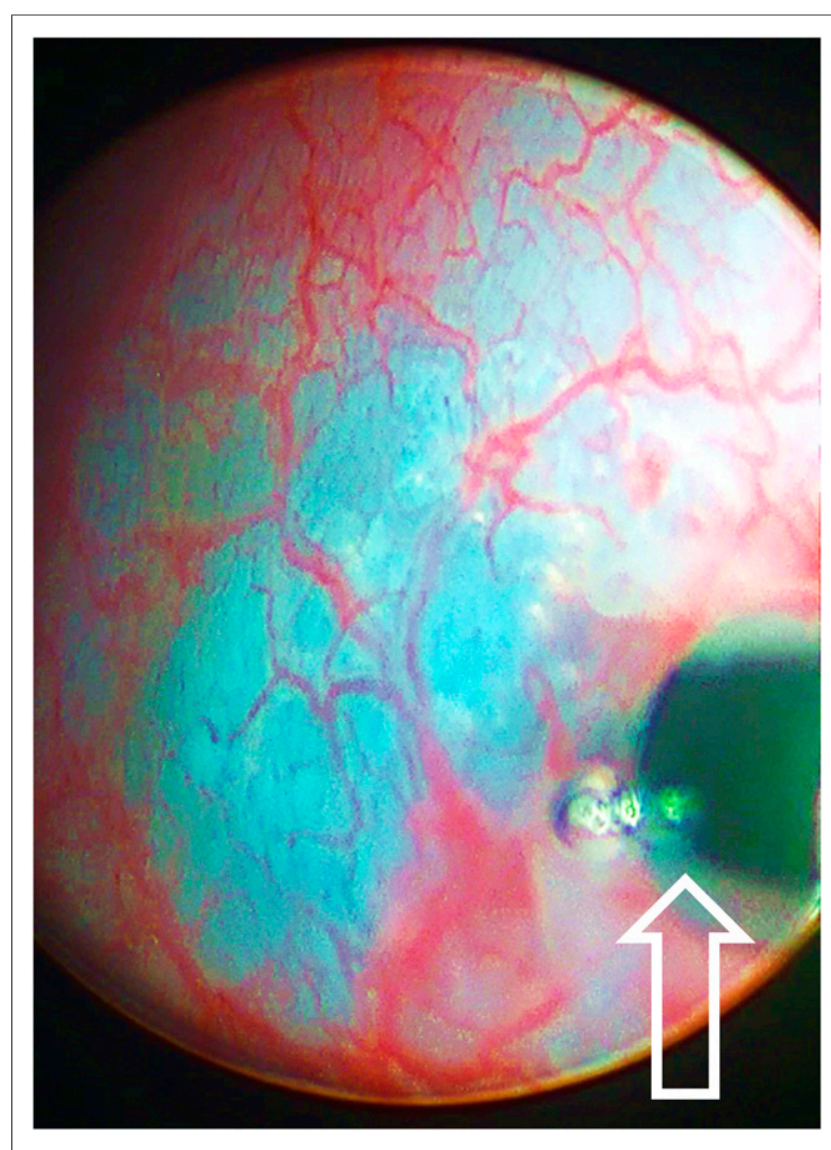

FIGURE 1. Cystoscopic injection (needle tip at arrow) into pig bladder of molecular imaging agent IRDye800CW-tilmanocept radiolabeled with ${ }^{68} \mathrm{Ga}$ and ${ }^{99 \mathrm{~m} T \mathrm{Tc} \text {. }}$

bladder cancer were used, limited and standard lymph node dissection missed positive LNs in $25 \%$ and $11 \%$ of patients, respectively (25). One controversy regarding PLND is the variety of templates with the lack of standardization and lack of real-time feedback of an adequate PLND for bladder cancer. The anatomic limits of dissection can vary greatly from surgeon to surgeon $(7,8,17,26)$, and from this standpoint tilmanocept can play a role in helping to identify at least the SLN and the first-order sites of metastasis, and standardize the dissection of these nodes. Moreover, this technique will allow the identification of high-risk LNs that may be located in aberrant locations, outside the standard dissection templates and allow the surgeon to extend the LN dissection for an adequate superextended lymph node dissection in some locally advanced disease.

On the basis of the PET/CT results, we will conduct the human trial using at least $9.3 \mathrm{MBq}(0.25 \mathrm{mCi})$ of ${ }^{68} \mathrm{Ga}$. The lowest ${ }^{68} \mathrm{Ga}$ dose used in the third pig study, 3.0 MBq $(80 \mu \mathrm{Ci})$, was not adequate to visualize both SLNs visualized by the FireFly camera. The right external iliac LN was fluorescent and qualified as an SLN on the basis of the ex vivo ${ }^{99 m}$ Tc activity measured by the $\gamma$-well-counter. Several factors could contribute to the inability of the PET/CT scanner to visualize the external iliac LN in the third study. The activity within the LN at $1 \mathrm{~h}$ after administration could have been too low; at $33 \mathrm{~h}$ after injection, the LN held $0.38 \%$ of the injected dose (Table 2), which based on a 3.0-MBq dose equaled $0.011 \mathrm{MBq}(0.3 \mu \mathrm{Ci})$. Additionally, coincidence photon scatter from the urinary bladder and injection site could reduce the ability to detect the activity in the LN. Tilmanocept was designed to clear the injection site by rapidly entering the lymphatic channels as well as blood capillaries. Consequently, at $1 \mathrm{~h}$ after an intramucosal injection, a high percentage of the injectate is circulating in the blood, resulting in accumulation by tissues that express the CD206 receptor, such as the liver, spleen, mesangium of the kidney, and bone marrow. Although it was disappointing not to have a $100 \% \mathrm{PET} / \mathrm{CT}$ detection rate, the regulatory agencies encourage the preclinical exploration of the lower dose limits pertaining to imaging sensitivity, which for a fluorescent-tag radiopharmaceutical pertains to the amount of fluorophore and radioactivity injected.

A phase 1 clinical trial would use the ${ }^{99 \mathrm{~m}} \mathrm{Tc}$ radiolabel; routine clinical application would use only 1 of the 2 radiolabels: ${ }^{68} \mathrm{Ga}$ for PET/CT or PET/MRI preoperative imaging or ${ }^{99 \mathrm{~m}} \mathrm{Tc}$ for SPECT/ CT imaging before the robotic surgery. Preliminary calculation of the radiation-absorbed dose indicates that the effective dose equivalent of a ${ }^{99 \mathrm{~m}} \mathrm{Tc}$ radiolabel is approximately half of an effective dose equivalent from ${ }^{68} \mathrm{Ga}$-labeled fluorescent tilmanocept. The preliminary dosimetry calculation assumed a biodistribution similar to ${ }^{99 \mathrm{~m} T c-t i l m a n o c e p t}$ after an intradermal injection of $1.0 \mathrm{nmol}$ (27). The MIRD calculation used the uterus as the injection site. A 9.3-MBq $(0.25 \mathrm{mCi})$ dose of ${ }^{68} \mathrm{Ga}$-labeled fluorescent tilmanocept would produce an absorbed radiation dose of approximately $0.002 \mathrm{cGy}$.

The current doctrine in the management of PLND for cystectomy is simply to remove as many LNs as possible from the pelvis at the time of surgery, because there is no a priori knowledge as to which LNs contain cancer during surgery (28). This dictum has continued and has benefited patients to improve overall survival

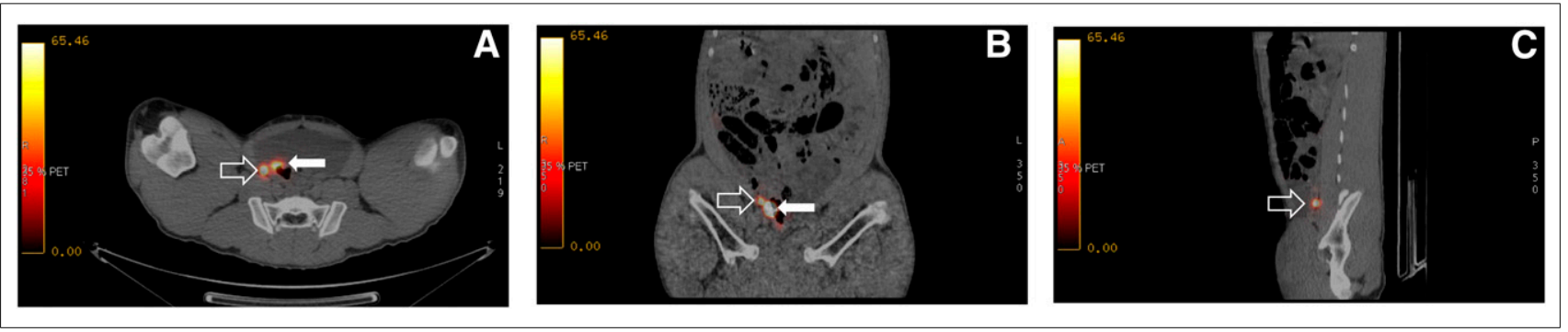

FIGURE 2. Fused PET/CT transaxial (A), coronal (B), and sagittal (C) cross-sections of right perivesicular LN (open arrow) adjacent to injection site in bladder wall (solid arrow). Injected dose of ${ }^{68} \mathrm{Ga}$ was $4.4 \mathrm{MBq}(120 \mu \mathrm{Ci})$; at time of PET/CT image this SLN contained $0.085 \mathrm{MBq}(2.3 \mu \mathrm{Ci})$ of radioactivity and had an SUV of 65.2. 


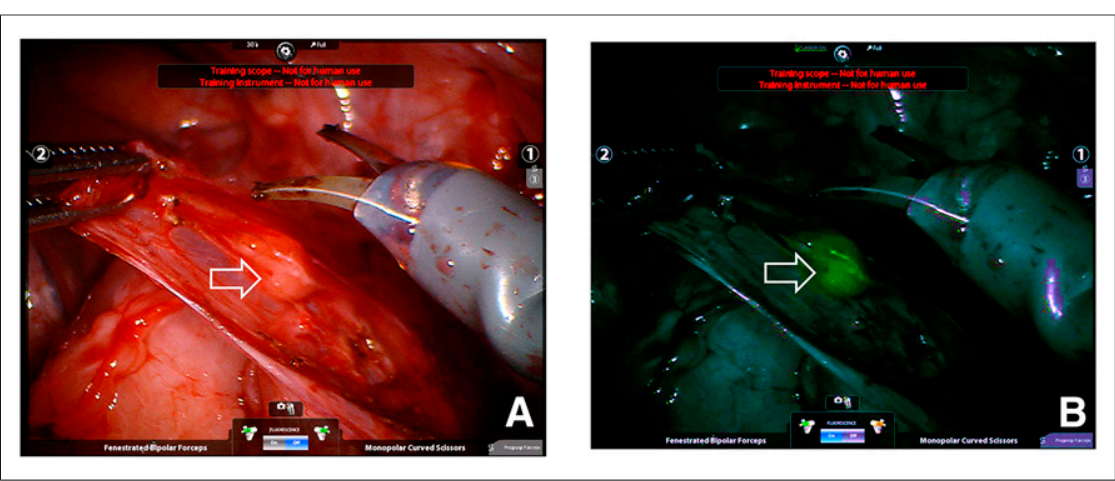

FIGURE 3. Intraoperative FireFly robotic surgical camera view looking into pelvis with a white light $(A)$ and same location with near-infrared view (B). Fluorescent SLN (open arrow) was visualized within minutes of starting mapping procedure. This SLN contained 88 pmol of fluorescent dye.

in the past $30 \mathrm{y}$ (7). However, the downside to this nonspecific method is the increased risk of complications, because extensive node dissection is more time consuming and can increase perioperative morbidity. It makes sense that by customizing the PLND, surgeons can minimize anesthesia time and intraoperative and major postoperative complications such as lymphoceles, lymphedema, vascular injury, deep venous thrombosis, and pulmonary embolus (29-31) while at the same time possibly increasing the chances of collecting the tumor-involved LNs.

Using fluorescent-labeled tilmanocept, we can further understand and learn more about SLN and lymphatic spread of metastasis in bladder cancer. In a lymphatic channel mapping study using ${ }^{99 \mathrm{~m}} \mathrm{Tc}$-labeled nanocolloid, the tracer was injected into 1 side of the bladder wall. However, $15 \%$ of nodes on the contralateral side were radioactive and contralateral lymphatic drainage was found in $40 \%$ of patients (32). This study demonstrated not just a simple direct ipsilateral lymphatic connection and spread, but a more complex lattice of lymphatic channels. Potential metastasis may be routinely missed if only a unilateral dissection was performed. Our limited understanding and study of the lymphatic drainage pattern and previous lack of dependable technology to identify SLNs have prevented the utility of limited or selective LN dissections for clinical outcomes. Ultimately, the goal is to limit the dissection of false-negative LNs to minimize complications without altering the current cancer-free and overall survival rates.

Advancement of the understanding of SLN in bladder cancer may provide new knowledge and improvements in clinical outcomes. Improvements can be made on technique of dissection to make sure no SLNs are left behind. In addition, the rate of skip metastasis in negative SLNs could be examined and allow a more selective lymph node dissection if the rate was negligible. Such an SLN mapping study has been tested in more than 1,000 prostatectomies, and only $1 \%$ of metastases were found in patients with negative SLNs and may improve detection over current guidelines for extended PLND (33). Conceivably in the future, with greater understanding of SLNs in MIBC, we can map SLNs in real time, dissect the SLN, and see whether cancer is present or not and make an intraoperative decision to perform or not perform a full extended lymph node dissection.

A limitation of a fluorescent-labeled radiopharmaceutical is that it requires an injection before cystectomy, which can create added cost, logistic problems, and also possible complications, which may delay the cystectomy. In addition, another limitation is that the agent is not specific to cancer and does not differentiate negative from positive LNs with metastatic spread. Nonspecific identification of only the SLN also can lead to problems of skip metastasis and the fundamental issue of leaving cancer behind. However, further studies are necessary to demonstrate the clinical significance of SLNs in MICB and improvements in complication rates after $\mathrm{LN}$ dissection.

With the advancement of the dual capabilities of tilmanocept, the standard treatment of MIBC can fundamentally change to accurately perform lymphadenectomy, rather than guessing the location of critical first-order SLNs. Our study will lead to future clinical trials and development of real-time image-guided interventions that can be delivered in a precise and safe manner, ultimately removing the guesswork for the surgeons and the burden of recurrences and complications from the MIBC survivor.

The measurements reported in Table 2 assume that the ${ }^{99 \mathrm{~m}} \mathrm{Tc}$ and fluorescent labels are stable for the $36 \mathrm{~h}$ during which the radiolabeled fluorescent tilmanocept resides in the SLN. We have studied the in vitro radiochemical stability of IRDye800CWtilmanocept when labeled with both ${ }^{68} \mathrm{Ga}$ and ${ }^{99 \mathrm{~m}} \mathrm{Tc}$ (16). We have also studied the in vivo stability of the covalent attachment of IRDye800CW to tilmanocept (15). In vitro, ${ }^{68} \mathrm{Ga}$ and ${ }^{99 \mathrm{~m}} \mathrm{Tc}$ remain attached to fluorescent tilmanocept after a 100 -fold dilution with an ethylenediaminetetraacetic acid-saline solution. Rapid dilution with a weak chelator (which can bind the metal for unique detection and analysis) is a standard method for testing the labeling stability of radiometals. The 100-fold dilution is far greater than the dilution that would occur after injection (from a $0.10 \mathrm{cc}$ volume) into the plasma of a mouse $(1.5 \mathrm{cc})$. We could not detect a loss of radiochemical purity after 11 -h incubations at $37^{\circ} \mathrm{C}$ in either isotonic saline or human plasma. We also demonstrated that the fluorescent signal from popliteal LNs remains unchanged immediately after injection (footpad) and at 24, 48, and $72 \mathrm{~h}$ after injection (15).

PET imaging will play an important role during the early phases of the development of fluorescent-labeled tilmanocept as a new molecular imaging agent. The quantitative capability of PET imaging will accomplish 3 goals. First, it will provide whole-body images of each patient, which will yield measurements of tissue distribution and the calculation of radiation-absorbed dose. Second, when preoperative imaging at different times after injection is performed, PET will permit the determination of the earliest time after injection that the intraoperative mapping can begin. Third, PET imaging is far more quantitative than fluorescence imaging with the currently available intraoperative camera systems. The PET images, when compared with the fluorescence images obtained during intraoperative mapping, may demonstrate that a real-time measurement of the fluorescence signal can be developed, providing a useful equivalent to the $10 \%$ rule, which is currently used during SLN mapping of breast cancer and melanoma. An early phase clinical trial without PET or SPECT imaging of tilmanocept would be problematic for the regulatory approval process, which may not accept a clinical trial that introduces 2 unproven entities - a new fluorescent molecular imaging agent and a detection system that was not designed or approved for quantitative measurements of fluorescence. 


\section{CONCLUSION}

${ }^{68} \mathrm{Ga}$-labeled fluorescent tilmanocept has logistic properties to obtain preoperative PET/CT and subsequent real-time intraoperative confirmation during robotic SLN dissection. Human clinical trials are needed to confirm the appropriate safety and subsequent clinical benefit of fluorescent-labeled tilmanocept.

\section{DISCLOSURE}

We acknowledge support by a Translational Research Award from the UCSD Moores Cancer Center (P30 CA023100) and the UCSD In Vivo Cancer and Molecular Imaging Program (P50 CA128346). Dr. David Vera is the inventor of Tilmanocept. No other potential conflict of interest relevant to this article was reported.

\section{REFERENCES}

1. Sherif A, Hasan MN, Marits P, Karlsson M, Winqvist O, Thorn M. Feasibility of T-cell-based adoptive immunotherapy in the first 12 patients with advanced urothelial urinary bladder cancer: preliminary data on a new immunologic treatment based on the sentinel node concept. Eur Urol. 2010;58:105-111.

2. Stein JP, Lieskovsky G, Cote R, et al. Radical cystectomy in the treatment of invasive bladder cancer: long-term results in 1,054 patients. J Clin Oncol. 2001;19: 666-675.

3. Dalbagni G, Genega E, Hashibe MIA, et al. Cystectomy for bladder cancer: a contemporary series. J Urol. 2001;165:1111-1116.

4. Gakis G, Efstathiou J, Lerner SP, et al. ICUD-EAU International Consultation on Bladder Cancer 2012: radical cystectomy and bladder preservation for muscleinvasive urothelial carcinoma of the bladder. Eur Urol. 2013;63:45-57.

5. Dhar NB, Klein EA, Reuther AM, Thalmann GN, Madersbacher S, Studer UE. Outcome after radical cystectomy with limited or extended pelvic lymph node dissection. J Urol. 2008;179:873-878.

6. Zehnder P, Studer UE, Skinner EC, et al. Super extended versus extended pelvic lymph node dissection in patients undergoing radical cystectomy for bladder cancer: a comparative study. J Urol. 2011;186:1261-1268.

7. Skinner DG. Management of invasive bladder cancer: a meticulous pelvic node dissection can make a difference. J Urol. 1982;128:34-36.

8. Bhindi B, Yu J, Kuk C, et al. The importance of surgeon characteristics in impacting oncologic outcomes in patients undergoing radical cystectomy. J Urol. 2014;192:714-719.

9. Vera DR, Wallace AM, Hoh CK, Mattrey RF. A synthetic macromolecule for sentinel node detection: ${ }^{99 m}$ Tc-DTPA-mannosyl-dextran. J Nucl Med. 2001;42: 951-959.

10. Emerson DK, Limmer KK, Hall DJ, et al. A receptor-targeted fluorescent radiopharmaceutical for multi-reporter sentinel lymph node imaging. Radiology. 2012;265:186-193.

11. Hosseini A, Baker JL, Tokin CA, et al. Fluorescent-tilmanocept for tumor margin analysis in the mouse model. J Surg Res. 2014;190:528-534.

12. Wallace AM, Han LK, Povoski SP, et al. Comparative evaluation of [ ${ }^{99 \mathrm{~m}} \mathrm{Tc}$ ] tilmanocept for sentinel lymph node mapping in breast cancer patients: results of two phase 3 trials. Ann Surg Oncol. 2013;20:2590-2599.

13. Sondak VK, King DW, Zager JS, et al. Combined analysis of phase III trials evaluating $\left[{ }^{99 \mathrm{~m}} \mathrm{Tc}\right]$ tilmanocept and vital blue dye for identification of sentinel lymph nodes in clinically node-negative cutaneous melanoma. Ann Surg Oncol. 2013;20:680-688.
14. Agrawal A, Civantos FJ, Brumund KT, et al. [ $\left.{ }^{99 \mathrm{~m}} \mathrm{Tc}\right]$ tilmanocept accurately detects sentinel lymph nodes and predicts node pathology status in patients with oral squamous cell carcinoma of the head and neck: results of a phase III multiinstitutional trial. Ann Surg Oncol. 2015;22:3708-3715.

15. Qin Z, Hall DJ, Liss MA, et al. Optimization via specific fluorescence brightness of a receptor-targeted probe for optical imaging and positron emission tomography of sentinel lymph nodes. J Biomed Opt. 2013;18:101315.

16. Qin Z, Hoh CK, Hall DJ, Vera DR. A tri-modal molecular imaging agent for sentinel lymph node mapping. Nucl Med Biol. 2015;42:917-922.

17. Liss MA, Kader AK. Robotic-assisted laparoscopic radical cystectomy: history, techniques and outcomes. World J Urol. 2013;31:489-497.

18. Liss MA, Stroup SP, Qin Z, Hall DJ, Kane CJ, Vera DR. Robotic-assisted fluorescent-guided sentinel lymph node dissection in a preclinical model. Urology. 2014;84:982.e9-14.

19. Stroup SP, Kane CJ, Farchshchi-Heydari S, et al. Preoperative sentinel lymph node mapping of the prostate using PET/CT fusion imaging and Ga-68-labeled tilmanocept in a dog model. Clin Exp Metastasis. 2012;29:673-680.

20. Liss MA, Farshchi-Heydari S, Qin Z, et al. Preclinical evaluation of roboticassisted sentinel lymph node fluorescence imaging. J Nucl Med. 2014;55:15521556.

21. Stein JP, Skinner DG. The role of lymphadenectomy in high-grade invasive bladder cancer. Urol Clin North Am. 2005;32:187-197.

22. Sherif A, De La Torre M, Malmstrom PU, Thorn M. Lymphatic mapping and detection of sentinel nodes in patients with bladder cancer. J Urol. 2001;166: 812-815.

23. Liedberg F, Chebil G, Davidsson T, Gudjonsson S, Mansson W. Intraoperative sentinel node detection improves nodal staging in invasive bladder cancer. J Urol. 2006;175:84-88.

24. Malmström PU, Ren ZP, Sherif A, de la Torre M, Wester K, Thorn M. Early metastatic progression of bladder carcinoma: molecular profile of primary tumor and sentinel lymph node. J Urol. 2002;168:2240-2244.

25. Dangle PP, Gong MC, Bahnson RR, Pohar KS. How do commonly performed lymphadenectomy templates influence bladder cancer nodal stage? J Urol. 2010;183:499-503.

26. Hellenthal NJ, Hussain A, Andrews PE, et al. Lymphadenectomy at the time of robot-assisted radical cystectomy: results from the International Robotic Cystectomy Consortium. BJU Int. 2011;107:642-646.

27. Wallace AM, Hoh CK, Ellner SJ, Darrah DD, Schulteis G, Vera DR. Lymphoseek: a molecular imaging agent for melanoma sentinel lymph node mapping. Ann Surg Oncol. 2007;14:913-921.

28. Fang AC, Ahmad AE, Whitson JM, Ferrell LD, Carroll PR, Konety BR. Effect of a minimum lymph node policy in radical cystectomy and pelvic lymphadenectomy on lymph node yields, lymph node positivity rates, lymph node density, and survivorship in patients with bladder cancer. Cancer. 2010;116:1901-1908.

29. Yuh B, Artibani W, Heidenreich A, et al. The role of robot-assisted radical prostatectomy and pelvic lymph node dissection in the management of high-risk prostate cancer: a systematic review. Eur Urol. 2014;65:918-927.

30. Ploussard G, Briganti A, de la Taille A, et al. Pelvic lymph node dissection during robot-assisted radical prostatectomy: efficacy, limitations, and complications-a systematic review of the literature. Eur Urol. 2014;65:7-16.

31. Lee HJ, Kane CJ. How to minimize lymphoceles and treat clinically symptomatic lymphoceles after radical prostatectomy. Curr Urol Rep. 2014;15:445-456.

32. Roth B, Zehnder P, Birkhauser FD, Burkhard FC, Thalmann GN, Studer UE. Is bilateral extended pelvic lymphadenectomy necessary for strictly unilateral invasive bladder cancer? J Urol. 2012;187:1577-1582.

33. Weckermann D, Dorn R, Trefz M, Wagner T, Wawroschek F, Harzmann R. Sentinel lymph node dissection for prostate cancer: experience with more than 1,000 patients. J Urol. 2007;177:916-920. 